\title{
AS CONSEQUÊNCIAS DA GRAVIDEZ NA ADOLESCÊNCIA EM UM MUNICIPIO DO OESTE PARANAENSE
}

\section{THE CONSEQUENCES OF PREGNANCY IN ADOLESCENCE IN A MUNICIPALITY OF THE WEST PARANAENSE}

\author{
Gilson Fernandes da Silva ${ }^{1 *}$, Simone Grein Borges² ${ }^{2}$ Angela Israel Graeff Borges \\ Capelete $^{3}$, Daisy Cristina Rodrigues ${ }^{4}$ \\ 1Enfermeiro. Gerente da Escola de Saúde Pública Municipal de Cascavel/PR. Coordenador e Tutor \\ do Programa de Residência Multiprofissional em Saúde da Família - Cascavel/PR. Mestre em \\ Biociências e Saúde pela UNIOESTE. ${ }^{2}$ Enfermeira. Egressa do curso de Enfermagem da \\ Universidade Paranaense - UNIPAR/Cascavel. ${ }^{3}$ Enfermeira Obstetra. Fundação Hospitalar São \\ Lucas. Mestranda em Biociências e Saúde pela Universidade Estadual do Oeste do \\ Paraná/UNIOESTE. ${ }^{4}$ Enfermeira. Mestre em Enfermagem/UFSM. Docente do Colegiado de \\ Enfermagem da UNIPAR/Cascavel. \\ * Autor correspondente: gilson enfermeiro@hotmail.com , https://orcid.org/0000-0001-7853-6284
}

DOI: $10.35984 / f j h . v 2 i 2.192$

\section{RESUMO}

Adolescência compreende o período entre 12 a 18 anos. É uma fase de mudanças rápidas e com início das atividades sexuais cada vez mais cedo, sem proteção, com risco de gravidez e (ISTs) infecções sexualmente transmissíveis. A gravidez precoce traz problemas tanto para as adolescentes quanto para a família. O objetivo foi identificar o perfil das consequências da gravidez na adolescência, os padrões da sexualidade, e a contracepção na adolescência. Tratou-se de uma pesquisa de campo, documental, exploratória, descritiva com análise qualiquantitativa. Foi aplicado questionário à 9 adolescentes grávidas entre 12 e 17 anos, participantes das reuniões de gestante, em Três Barras do Paraná, nos meses de junho e julho de 2017. Os resultados obtidos através dessa pesquisa demonstraram: faixa etária de maior prevalência 16 a 17 anos 5 (55.56 \%) e ensino fundamental incompleto 3 (34\%). Esperase contribuir com discussões sobre gravidez na adolescência, métodos contraceptivos, proporcionando conhecimento, acesso e desenvolvimento de estratégias e projetos.

Palavras-Chave: Gravidez na Adolescência, Contracepção, Sexualidade.

\begin{abstract}
Adolescence comprises the period between 12 to 18 years. It is a phase of rapid changes and with the beginning of sexual activities earlier and earlier, without protection, with risk of pregnancy and sexually transmitted infections (STIS). Early pregnancy poses problems for both adolescents and the family. The objective was to identify the profile of the consequences of teenage pregnancy, sexuality patterns, and teenage contraception. It was a field research, documentary, exploratory, descriptive with qualitative and quantitative analysis. A questionnaire was applied to 9 pregnant teenagers between 12 and 17 years old, participating in the pregnant women meetings, in Três Barras do Paraná, in the months of June and July 2017. The results obtained through this research showed: age group with the highest prevalence 16 to 17 years 5 (55.56\%) and incomplete elementary school 3 (34\%). It is expected to contribute to discussions about teenage pregnancy, contraceptive methods, providing knowledge, access and development of strategies and projects.
\end{abstract}

Keywords: Pregnancy in Adolescence, contraception, Sexuality. 


\section{INTRODUÇÃO}

A adolescência compreende o período entre 12 a 18 anos, podendo variar com as características de personalidade ou até mesmo a experiência de vida de cada um (SANTOS; CARVALHO, 2006). Conforme o Estatuto da Criança e do Adolescente, Lei no 8069/90, de 13 de julho de 1990, adolescência compreende o período entre 12 a 18 anos (LOPES, 2013) e para a Organização Mundial de Saúde (OMS) de 10 a 19 anos (SANTOS, et al., 2010). Essa é uma fase de muitas mudanças no ciclo de vida e acontece rapidamente, sendo considerada uma transição da vida infantil para a vida adulta. (TABORDA, et al., 2014).

A gravidez na Adolescência vem sendo tratado como um problema de saúde pública, tanto por trazer riscos para a criança gerada quanto para a adolescente gestante (SANTOS, et al., 2010). Em estudo realizado com adolescentes brasileiros diz que esses tem iniciado a vida sexual cada vez mais cedo e com maior número de parceiros (SANTOS, et al., 2010). Apesar de essa situação ocorrer em todas as classes sociais, ainda assim ocorre em maior parte nas classes menos favorecidas e com baixa escolaridade. Outros fatores como a diminuição da idade da menarca e a primeira relação sexual colaboram para o aumento dessas taxas (LOPES, 2013).

Sabe-se que as adolescentes são fisiologicamente imaturas para passar por uma gestação, além dos riscos perinatais serem maiores nessa faixa etária, principalmente se essa gestação acontecer com menos de dois anos após a menarca ou com idade menor de 15 anos (PRETTO, et al., 2016). Essas adolescentes sentem medo, insegurança, desespero e solidão principalmente no momento em que descobrem a gravidez. Porém vale ressaltar que nem toda gravidez adolescente é indesejada, algumas são por desejo próprio, planejamento e/ou decorrentes de uma vida afetiva estável (TABORDA, et al., 2014).

Uma gestação não planejada significa que houve exposição de pelo menos uma vez a situação de risco, relação sexual sem proteção, ou seja, sem o uso de preservativo (SANTOS, et al., 2010). Lembrando que essa situação repercute negativamente nos índices de evasão escolar antes e após a gestação (TABORDA, et al., 2014).

Aos serviços de saúde cabe a prestação de uma assistência adequada e o desenvolvimento de ações educativas que abordem a sexualidade com informações claras e científicas, mostrando as diferenças culturais do início da atividade sexual e reprodutiva, a fim de mostrar maiores informações, mais conhecimento e que esses sejam resolutivos (BRASIL, 2011).

Algumas pesquisas trazem fatores que possam determinar o risco da gravidez na adolescência, o início das atividades sexuais precoce, a deficiência de informações sobre métodos contraceptivos e a suposta falta de apoio a essas adolescentes (SANTOS, et al., 2010). De acordo com Patias e Dias (2011), apesar de todas as informações existentes sobre os métodos contraceptivos, cerca de 45 a $60 \%$ dos adolescentes brasileiros iniciam suas atividades sexuais sem o uso de algum método contraceptivo.

Estudos mostram que os adolescentes não fazem uso das informações sobre os métodos contraceptivos, por apresentarem dúvidas sobre os mesmos, por essas informações estarem incompletas para eles, por associarem isso a ideias erradas sobre a sexualidade e contraceptivos, e que a comunicação entre os pais e filhos a respeito dessa temática também é falha e incompleta, sendo prejudicada pela falta de intimidade entre eles. Além disso, o receio de fazer o uso incorreto desses métodos, desconhecimento, dificuldade de compra e os pedidos de provas de amor por parte FAG Journal of Health - ISSN 2674-550X, 2020, v.2, n.2, p. 187 
do parceiro para o não uso dos contraceptivos contribui para a relação desprotegida (PATIAS; DIAS, 2011). Nesse contexto o objetivo dessa pesquisa foi identificar o perfil das consequências da gravidez na adolescência, os padrões da sexualidade, e a contracepção na adolescência.

\section{METODOLOGIA}

Tratou-se de uma pesquisa de campo, documental, exploratória e descritiva com análise qualiquantitativa. Esse estudo foi desenvolvido na Unidade Básica de Saúde Central, durante a reunião de gestantes, na cidade de Três Barras do Paraná, conforme estatísticas do IBGE (2010), a população total era de 12.242 habitantes, sendo que 5.830 mulheres e aproximadamente 1.120 adolescentes. A população participante foram as gestantes adolescentes de 12 a 18 anos, que aceitaram participar da pesquisa e responder as questões solicitadas.

Foram usados como critério de inclusão nesse estudo, adolescentes com idade entre 12 a 18 anos, que aceitaram participar da pesquisa. E como critério de exclusão aquelas com idade inferior a 12 anos, superior a 18 anos ou aquelas adolescentes grávidas que não tinham condições de responder ao questionário da pesquisa por outras causas. Foi utilizada como instrumento para coleta dos dados uma entrevista com questões abertas e fechadas. Encaminhado uma cópia do projeto de pesquisa a instituição onde foi realizada a coleta de dados, conforme a declaração de permissão para uso de dados, essa pesquisa foi aprovada pelo juizado da Infância, e posteriormente foi encaminhado ao Comitê de Ética e Pesquisa, sendo aprovado em: 29 de maio de 2017, parecer no: 2.086.778 e CAEE no: 68393617.7.0000.0109.

A pesquisa foi desenvolvida segundo as diretrizes e normas regulamentadoras de pesquisa envolvendo seres humanos, a Resolução do Conselho Nacional de Saúde no 466/2012 e a Resolução 510/ 2016 que dispõe das Diretrizes e Normas regulamentadoras de Pesquisa Envolvendo Seres Humanos (BRASIL, 2012a; BRASIL, 2016a). Sendo assim como forma de preservar o anonimato, os participantes da pesquisa foram identificados por siglas: F1, F2, F3, F4, F5, F6, F7, F8 e F9.

\section{RESULTADOS E DISCUSSÃO}

Apresentamos os dados obtidos por meio da pesquisa realizada na cidade de Três Barras do Paraná, em uma Unidade Básica de Saúde Central, no período de junho e julho de 2017, durante a reunião de gestante que ocorre mensalmente. A coleta foi realizada com 9 adolescentes. Na figura 1 apresentamos a distribuição das adolescentes conforme a faixa etária, sendo que de 12 a 13 anos foram 2(22.22\%), de 14 a 15 anos 2(22.22\%), de 16 a 17 anos foram 5(55.56\%) adolescentes grávidas. Evidenciou-se que a faixa etária de maior incidência de gravidez é de 16 a 17 anos.

Para Chalem, et al., (2007), a média de idade das adolescentes é de 17 anos, variando de 11 a 19 anos; $17 \%$ de até 15 anos. A idade média das adolescentes gravidas é de 17,3 anos; e de 18,5\% com 13 e 15 anos (VIEIRA, et al., 2017).

Segundo Vieira, et al., (2017), no Brasil a gestação das adolescentes entre 15 e 19 anos cresceu até o fim do século XX, vindo a declinar no início do século XXI. Porém, mesmo com esta recente queda, pode-se afirmar que continuam a ocorrer casos de gravidez na adolescência no País. Foram registrados 559.991 nascimentos de mães com menos de 19 anos em 2013, o que reforça a importância de estudos sobre o tema. 
1900ral

1900ral

1900ral

1900ral

1900ra

1900ral

1900ral

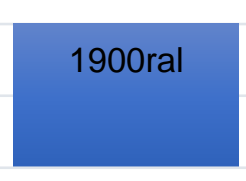

$12-13$ anos

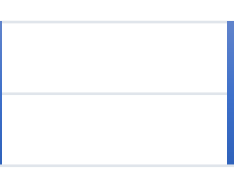

$14-15$ anos

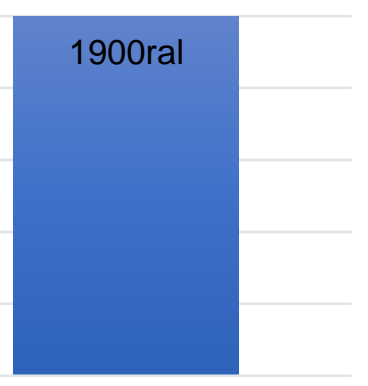

$16-17$ anos

Figura 1. Distribuição das adolescentes de acordo com a faixa etária.

Para Moreira, et al., (2008), no Brasil estima-se que nascem a cada ano um milhão bebês de mães com idade entre 10 a 19 anos, o que corresponde a $20 \%$ do total de nascidos vivos no País. Estudos trazem que além dos crescentes números, a faixa etária está cada vez menor em adolescentes que engravidam, isso chama a atenção da sociedade e do governo, mundialmente, gerando a necessidade de programas de atuação na saúde pública com ampla cobertura e envolvimento de vários profissionais de saúde, dentre eles, o enfermeiro (MOREIRA, et al., 2008).

$\mathrm{Na}$ figura 2 apresentamos a distribuição das adolescentes conforme o nível de escolaridade, sendo essas com o ensino fundamental incompleto foram $3(34 \%)$, ensino fundamental completo $1(11 \%), 1^{\circ}$ grau incompletos $3(33 \%), 1^{\circ}$ grau completo $1(11 \%)$ e com o $2^{\circ}$ grau completo somente $1(11 \%)$. Evidenciou-se que o grau de escolaridade com maior incidência foi o ensino fundamental incompleto e ensino fundamental completo.

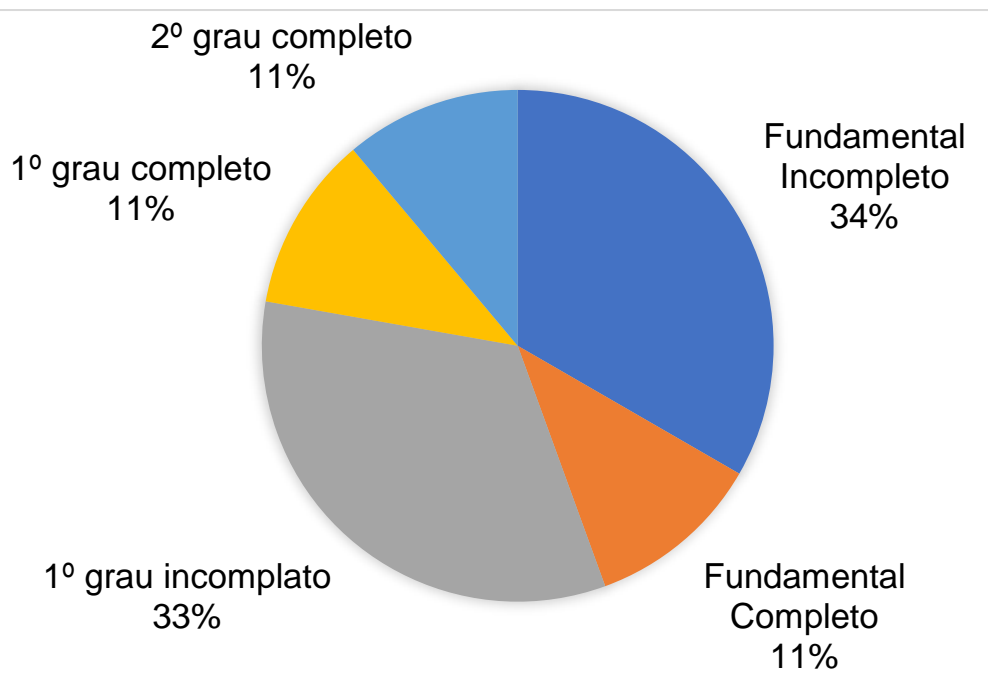

Figura 2. Distribuição das adolescentes conforme o nível de escolaridade.

Para Taborda, et al., (2014), a gestação precoce pode trazer desvantagens a fase educacional da adolescente gestante, contribuindo para a evasão escolar e dificultando o retorno aos estudos, limitando o seu ingresso acadêmico e as possibilidades de entrar para o mercado de trabalho. 
Estudos trazem que quanto maior o grau de instrução das adolescentes, maior a possibilidade de negociação de um método contraceptivo e seu uso correto, tanto na primeira quanto nas próximas relações sexuais (NERY et al., 2015). Apesar de essa situação ocorrer em todas as classes sociais, ainda assim ocorre em maior parte nas classes menos favorecidas e com baixa escolaridade (LOPES, 2013).

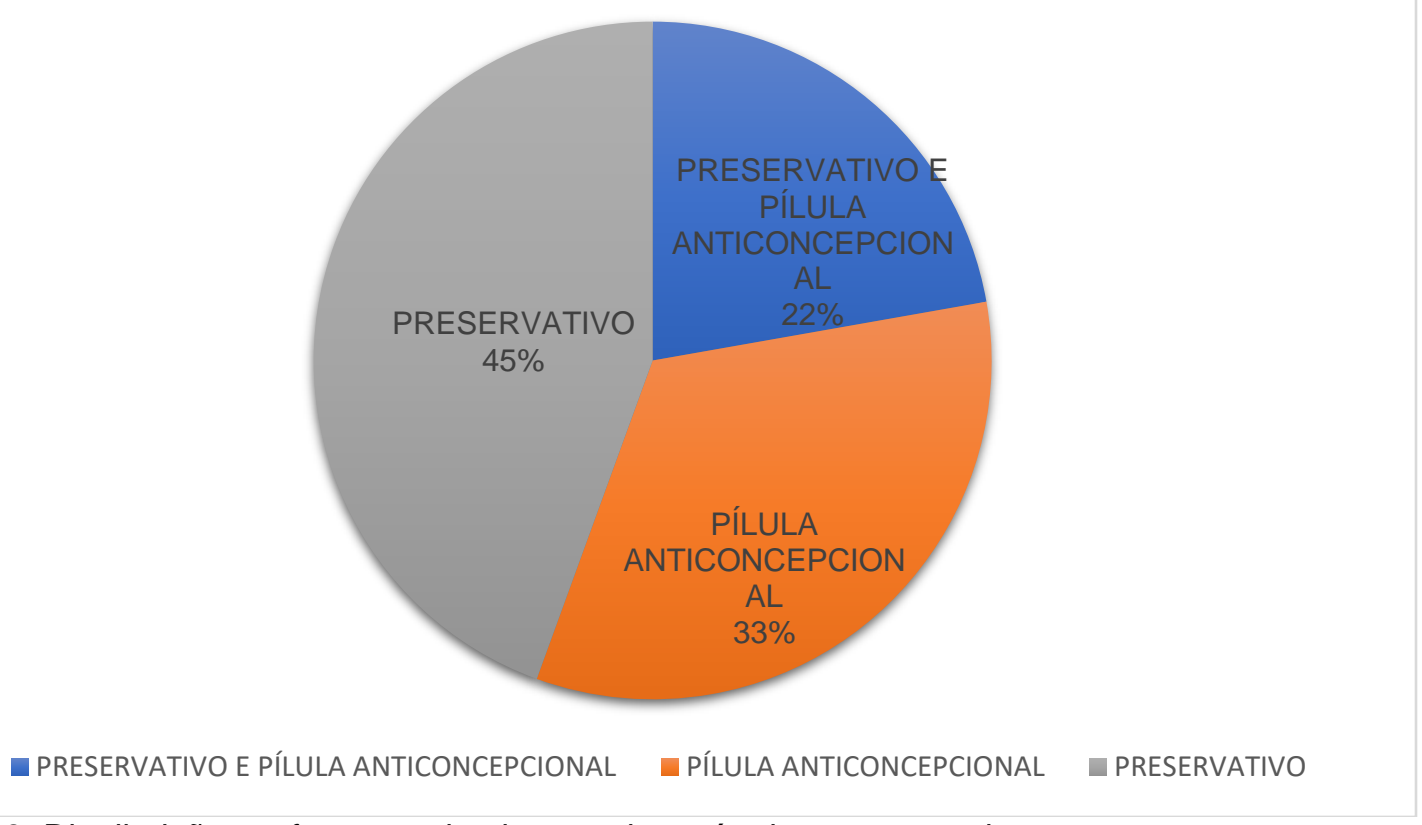

Figura 3. Distribuição conforme conhecimento dos métodos contraceptivos.

$\mathrm{Na}$ figura 3 apresentamos a distribuição das adolescentes conforme conhecimento de método contraceptivo. Disseram que conhecem preservativo e pílula anticoncepcional 2(22\%), que conhecem apenas pílula anticoncepcional 3(33\%) e que conhecem somente preservativo 4(45\%). Evidenciou-se que o método contraceptivo mais conhecido pelas adolescentes foi o preservativo. Para Santos e Nogueira (2009), a utilização de métodos contraceptivos não é eficaz na adolescência, embora grande parte deles conheçam os métodos mais comuns, como a camisinha e a pílula anticoncepcional, as razões tal comportamento seria a imaturidade psicoemocional, característica da adolescência.

Estudos trazem que o conhecimento dos adolescentes sobre métodos contraceptivos mais conhecidos e usados são o anticoncepcional oral $94,2 \%$ e a camisinha 91,7\% (SANTOS; NOGUEIRA, 2009). Para Taborda, et al., (2014), as adolescentes conhecem meios de evitar uma gravidez, porém, muitas vezes se recusa a usar, pois assim terá que assumir a vida sexual. Assim são comuns os pensamentos em relação à contracepção: se eu "tomar anticoncepcional meu corpo irá se transformar, e denunciar minha vida sexual". Segundo Dadoorian (2003), as adolescentes afirmaram saber que praticar atividade sexual sem 0 uso de contraceptivos poderia provocar uma gravidez. Porém, relataram que não usaram nenhum método no início da vida sexual. Esse dado questiona o fato de que a gravidez na adolescência ocorre por falta de informação sexual. Algumas adolescentes vão ao ginecologista com suas mães, mas mesmo assim elas optam por não usar os métodos contraceptivos.

Quando questionadas sobre o que mudou em sua vida desde a gravidez? Quais seus planos para o futuro? Obtivemos as seguintes respostas: 
F1- Mudou tudo, o jeito de pensar, jeito de agir. Fazer uma poupança em seu nome para ter a garantia para o futuro do nosso bebê.

F2- Não mudou nada.

F3- Rotina, mudou o convívio com os amigos, parei de ir a festas noturnas, parei de beber bebida alcoólica. Daqui alguns meses arrumar um emprego e iniciar uma faculdade de administração.

F4- Algumas coisas, rotina, cuidados, entre outros.

F5- Muitas coisas mudaram em minha vida, antes eu não me preocupava com coisas que hoje são muito importantes para mim. Meus planos para o futuro é estudar, me formar e pensar no futuro e no bem da minha vida e principalmente em minha filha.

F6- Futuro: Trabalhar, estudar e cuidar da minha filha, e nada mudou.

F7- Mudou tudo, tive consultas mais frequentes, cuidados com a comida, chás e remédios. Trabalhar para ter condições para cuidar da minha filha da melhor forma.

F8- Mudou tudo.

F9- Mudou tudo, cuidar do meu bebê, dar carinho a ele ou ela, e ficar com meu companheiro pra nóis cuidar juntos.

Todas as transformações que ocorrem física e psicologicamente com as adolescentes podem se revelar em mudanças biológicas, do aprendizado, comportamento, de descobertas, interação, socialização e de inúmeros outros processos. Sabe-se que a gravidez na adolescência gera consequências no emocional dessas jovens (TABORDA, et al., 2014). Para Taborda, et al., (2014) são muitas as consequências da gravidez na adolescência, essas podem prejudicar vários aspectos da vida e do bem-estar dessas jovens, de seus filhos e de sua família. Quando questionadas sobre como sua família encarou sua gravidez? Surgiram as seguintes respostas:

F1- Com espanto, mas aceitaram bem.

F2- Foi difícil.

F3- No começo foi meio difícil por causa da minha idade, eu não era casada ainda, mas agora estão aceitando numa boa, agora estão adorando a ideia.

F4- Por não ter sido algo planejado e nem algo esperado, foi um choque no começo. Porém a família aceitou.

F5- No começo foi um pouco difícil de lidar com tudo, mas agora está tudo bem, todos me ajudam e cada dia mais está me ajudando mais.

F6- Normal.

F7- Normal, pois o que eles mais queriam era serem avós.

F8- Levou numa boa.

F9- Normal. 
Segundo Taborda, et al., (2014), apesar do choque e decepção e revolta de alguns pais, como passar do tempo acabam aceitando toda a situação. As reações da família com a notícia da adolescente estar grávida tendem a ser contraditórias, sendo comum o de sentimento de revolta, abandono e aceitação do "inevitável" (SANTOS; NOGUEIRA, 2009). Para Dias e Teixeira (2010), as dificuldades, inseguranças e falta de habilidade com a maternidade e o pouco conhecimento sobre desenvolvimento infantil que as adolescentes possuem, podem se transformar em risco para o desenvolvimento do bebê. Contudo estudos mostram que, se a jovem recebe apoio, ela pode superar tais dificuldades.

Para Riekowski e Almeida (2009), as reações da família frente a adolescente grávida tendem a ser contraditórias, sendo comum o sentimento de revolta, abandono e aceitação do "inevitável". No início, a rejeição e o constrangimento podem levar a família a tomar atitudes radicais, como, expulsar a adolescente de casa, induzir ou forçar o aborto e impor responsabilidades, exigindo o casamento ou a união estável.

Pode- se observar a reação das famílias das adolescentes diante de uma gravidez varia de acordo com a classe social. Nas classes populares as famílias apresentam melhor aceitação do fato, principalmente a mãe e a avó, diferente das famílias das adolescentes de classe média, que não desejam a gravidez das filhas adolescentes (DADOORIAN, 2003).

\section{CONSIDERAÇÕES FINAIS}

A adolescência é um processo natural da vida, período em que ocorre grande parte das transformações de um ser humano, transformações essas hormonais, físicas, psicológicas e comportamentais. Sabemos que os profissionais de saúde têm papel fundamental na inserção de ações de prevenção e promoção da saúde e de ações educativas, a fim de melhorar o conhecimento dos adolescentes sobre gravidez e os meios de evita-las e traze-los para perto adquirindo vínculo com os mesmos. $O$ enfermeiro na realização de suas atribuições precisa acompanhar esses adolescentes, desenvolver vínculo, prestar assistência adequada e de qualidade, desenvolver ações educativas que abordem a sexualidade e os meios contraceptivos de forma clara e científica, abordando as diferenças do início da atividade sexual e reprodutiva, a fim transmitir informações e conhecimentos que tenham resolubilidade.

As ações de educativas visam diminuir os casos de gravidez na adolescência e incentivar os mesmos a usarem meios contraceptivos e preservativos, pois, é a forma de evitar as infecções sexualmente transmissíveis e buscarem informações caso haja dúvidas.

Este estudo além de nos trazer as consequências da gravidez na adolescência, destacou a necessidade de desenvolver atividades no município para que haja uma diminuição desses números que se tornam crescentes, sendo necessárias ações como educação continuada para esses adolescentes, roda de conversa, palestras nas escolas, proporcionar vínculo com a equipe multiprofissional da Unidade de Saúde e os adolescentes, visando assim um acompanhamento adequado, visando à melhoria dos indicadores de saúde. Todos os levantamentos obtidos com essa pesquisa nos encaminham para ações que venham a reduzir esses os índices de gravidez na adolescência. Espera-se que esse estudo possa contribuir e fomentar discussões na comunidade acadêmica sobre a temática e oportunizando o desenvolvimento de estratégias para minimizar as consequências da gravidez na adolescência. 


\section{REFERÊNCIAS}

BRASIL. Ministério da Saúde. Política Nacional de Atenção Integral à Saúde da Mulher: Princípios e Diretrizes. Brasília - DF: 2011.

BRASIL. Ministério da Saúde. Resolução № 466, de 12 de dezembro de 2012. Brasília, 2012a. Aprova as normas regulamentadoras de pesquisas envolvendo seres humanos. Disponível em: http://conselho.saude.gov.br/resolucoes/2012/Reso466.pdf. Acesso em: 19 mar. 2017.

BRASIL. Ministério da Saúde. Resolução № 510, de 07 de abril de 2016. Brasília, 2016. Dispõe sobre as normas aplicáveis a pesquisas em Ciências Humanas e Sociais. Disponível em: http://conselho.saude.gov.br/resolucoes/2016/Reso510.pdf. Acesso em: 19 mar. 2017.

CHALEM, E. et al., Gravidez na adolescência: Perfil sociodemográfico e comportamental de uma população da periferia de São Paulo, Brasil. Cadernos de Saúde Pública. Rio de Janeiro. 2007. Disponível em: http://www.scielo.br/scielo.php?script=sci_arttext\&pid=S0102-311X2007000100019. Acesso em: 25 ago. 2017.

DADOORIAN, D. Gravidez na adolescência: um novo olhar. Psicologia Clínica e Psicopatologia. Brasília/DF. $2003 . \quad$ Disponível em: http://www.scielo.br/scielo.php?script=sci_arttext\&pid=S1414$98932003000100012 \& \operatorname{lng}=$ pt\&tlng=pt. Acesso em: 26 ago. 2017.

DIAS, A. C. G; TEIXEIRA, M. A. P. Gravidez na Adolescência: Um olhar sobre um Fenômeno Complexo. Departamento de Psicologia. Santa Maria. RS, 2010. Disponível em: http://www.scielo.br/pdf/paideia/v20n45/a15v20n45.pdf. Acesso em: 29 mar. 2017.

IBGE. Instituto Brasileiro de Geografia e Estatística. Cidades. Três Barras do Paraná, censo 2010. População total, feminina e adolescente. Disponível em: http://www.cidades.ibge.gov.br/v3/cidades/municipio/4127858/pesquisa/23/2010.

Acesso em: 02 abr. 2017.

LOPES, A. C. B. Proteção Integral de Crianças e Adolescentes: Instrumentos Normativos Nacionais e Internacionais. Curitiba/PR. SECS. 2013.

MOREIRA, T. M. M. et al., Conflitos vivenciados pelas adolescentes com a descoberta da gravidez. Rev. Esc. Enferm. São Paulo. 2008. Disponível em: http://www.scielo.br/scielo.php?script=sci_arttext\&pid=S0080-62342008000200015. Acesso em: 27 ago. 2017.

NERY, I. S. et al., Fatores associados à reincidiva de gravidez após gestação na adolescência do Piaui, Brasil. Epidemiol. Serv. Saúde, Brasília. 2015. Disponível em: http://www.scielo.br/scielo.php?script=sci_arttext\&pid=S2237-

96222015000400671\&lng=en\&nrm=iso\&tlng=pt. Acesso em: 05 ago. 2017. 
PATIAS, N. D; DIAS, A. C. G. Fatores que Tornam Adolescentes Vulneráveis à Ocorrência de Gestação. Adolescência e Saúde. Rio de Janeiro. 2011. Disponível em: http://www.adolescenciaesaude.com/detalhe artigo.asp?id=272. Acesso em: 04 abr. 2017.

PRETTO, A.D. B. et al., Fatores Associados ao Baixo Peso ao Nascer entre Filhos de Mães Adolescentes. Adolescência e Saúde. v. 13, supl. 2. p. 139-149. Rio de Janeiro. 2016. http://www.adolescenciaesaude.com/detalhe artigo.asp?id=594. Acesso em: 25 mar. 2017.

RIEKOWSKI, T; ALMEIDA, V. Influência do Sistema Familiar na Repetição Indesejada da Gravidez na Adolescência. Ágora: Rev. Divulg. Cient, Mafra, v. 16, n. 2, 2009. Disponível em: http://www.periodicos.unc.br/index.php/agora/article/viewFile/29/144. Acesso em: 19 ago. 2017.

SANTOS, A.D; CARVALHO, A. V. D. Gravidez na Adolescência: Um Estudo Exploratório. Departamento de Psicologia. Maringá/PR. 2006. Disponível em: http://pepsic.bvsalud.org/scielo.php?script=sci arttext\&pid=S0006-

59432006000200002. Acesso em: 16 mar. 2017.

SANTOS, C. A. C. D; NOGUEIRA. K. T. Gravidez na Adolescência: Falta de informação? Adolescência e Saúde. Rio de Janeiro, 2009. Disponível em: http://www.adolescenciaesaude.com/detalhe artigo.asp?id=42. Acesso em: 30 mar. 2017.

SANTOS, E.C; et al., Gravidez na Adolescência: Análise Contextual de Risco e Proteção. Departamento de Psicologia. Maringá/PR- 2010. Disponível em: http://www.scielo.br/pdf/pe/v15n1/a09v15n1.pdf. Acesso em: 17 mar. 2017.

TABORDA, J. A; et al., Consequências da Gravidez na Adolescência para as Meninas Considerando as Diferenças Socioeconômicas entre elas. Caderno de Saúde Coletiva. Rio de Janeiro, 2014.2 Disponível em: http://www.scielo.br/pdf/cadsc/v22n1/1414-462X-cadsc-22-01-00016.pdf. Acesso em: 16 mar. 2017.

VIEIRA, E. M. et al., Gravidez na adolescência e transição para a vida adulta em jovens usuárias do SUS. Rev. Saúde Pública. 2017. Ribeirão Preto- SP. Disponível em:

http://www.scielo.br/pdf/rsp/v51/pt_0034-8910-rsp-S151887872016050006528.pdf. Acesso em: 26 ago. 2017. 